\title{
In vitro Picosecond Ultrasonics in a Single Cell
}

\author{
Bertrand AUDOIN ${ }^{1}$, Clément ROSSIGNOL ${ }^{1}$, Nikolay CHIGAREV ${ }^{1}$, Mathieu \\ DUCOUSSO, Marie-Christine DURRIEU ${ }^{2}$, Fabien GUILLEMOT ${ }^{2}$, \\ Guillaume FORGET ${ }^{2}$ \\ ${ }^{1}$ Laboratoire de Mécanique Physique, UMR CNRS 5469, Université Bordeaux 1, \\ 351 cours de la Libération, 33405 Talence, France \\ ${ }^{2}$ Laboratoire de Biomatériaux et Réparation Tissulaire, INSERM U 577, \\ Université Bordeaux 2, 146, rue Léo-Saignat, 33076 Bordeaux, France
}

\begin{abstract}
The picosecond ultrasonic technique is applied for the non-invasive evaluation of sound velocity at a submicron scale in living onion cells. Velocity and attenuation of hypersound in cells are measured by a femtosecond laser pump-probe technique. A nanometric co-polymer layer deposited between the cell and the substrate has been used to improve the photoacoustic signal. Comparison of the measured signals with the photoacoustic responses calculated according to thermoelastic generation mechanism and reflectometric detection shows high sensitivity to the cell adhesion on substrate. Measurements achieved in different vegetal cells illustrate the sensitivity of the technique. In addition to single cell imaging with the high lateral resolution provided by optics (ie $\approx 1 \mu \mathrm{m}$ ), the sensitivity of the measurements to cell compressibility suggests promising perspectives in the field of biology
\end{abstract}

Keywords: Picosecond opto-acoustics, single cell imaging, elastography at a sub-cell scale

\section{Introduction}

The picosecond ultrasonic technique [1] was developed during last 20 years due to permanent interest in the study of mechanical and thermal properties of structures of metals and semiconductors in nano and micro domains used essentially in solid state physics and microelectronics. This technique relies on generation and detection of nanometric ultra-short acoustic waves by the use of femtosecond laser pulses and it is based on the well-known pump-probe technique. Important advantages are the absence of direct contact to the sample and very high frequency range. The bandwidth is limited only by the dynamics of the photoacoustic response of the medium under study and/or by the duration of the laser pulse. The present paper demonstrates that picosecond ultrasonics techniques can be used to measure the mechanical properties of single alive cells, which are related to their interfacial and environmental surrounding.

\section{Samples and experimental set-up}

In this paper, the picosecond acoustic technique is applied to the non-invasive evaluation of ultrasound velocity and attenuation in the vacuole of alive single Allium cepa cells (common onion). Most mature plant cells have one or several vacuoles that can occupy as much as $90 \%$ of the volume for certain cell types and conditions (the case of the Allium cepa cells).

Slices of alive cells are deposited directly on the surface of a polished titanium alloy (Ti6Al4V) substrate. The thickness and the lateral size of a cell are measured by optical microscopy as $\sim 5 \mu \mathrm{m}$ and $50-100 \mu \mathrm{m}$, respectively. In addition, another polished titanium alloy substrate is coated by an optically transparent $0.25 \mu \mathrm{m}$ layer made of 
polyethyleneimine (PEI) as a model molecule [2]. All the experiments are performed at the room temperature of $21^{\circ} \mathrm{C}$ without any treatment or fixation of the alive cells.

Well known reflectometric pump-probe experimental set-up is used [1] to generate and detect acoustic waves in the cells. The radiation of a femtosecond laser (wavelength $0.795 \mu \mathrm{m}$, pulse duration $100 \mathrm{fs}$, energy of the pulse $10 \mathrm{~nJ}$ ) is divided in two beams to provide the pump and probe beams. An optical delay line (0-12 ns) is introduced in the probe beam path. The pump beam is modulated at a frequency of $300 \mathrm{kHz}$ to provide a reference signal for lock-in amplification. Intensity of the reflected probe beam is detected by a photodiode. Such a set-up allows the detection of reflectivity relative variations at the level as low as $10^{-7}$. Both beams are focused on the sample surface by a same objective with a 50x magnification. The width at mid height of the space crosscorrelation of the pump and probe beams is approximately $2 \mu \mathrm{m}$.

\section{Hypersound generation and detection}

Let us now consider a transparent biological cell on the surface of an optically polished metallic substrate (Fig. 1). The pump and probe beams are focused on the substrate through the cell. Absorption of the pump laser beam in the substrate provides a local change of the temperature, which induces thermal and acoustic strains. The so generated sound pulse propagates in the cell and in the substrate. The strain field induces a transient local change in the optical index of the cellular vacuole propagating with longitudinal sound velocity. This change provides a second reflection of the probe beam scattered from the acoustic pulse and interfering on the detector with the beam reflected from the interface with the metallic substrate. So called Brillouin scattering oscillations are the result of this interference. Their frequency at normal incidence of probe beam is[3]:

$$
f_{B}=\frac{2 n v}{\lambda}
$$

where $\lambda$ is the wavelength of probe light, and $v$ is the velocity of sound propagation in the medium. If the optical index $n$ of the medium is known it is straightforward to obtain the value of sound velocity from relation (1). The coefficient of sound attenuation $\alpha$ at the frequency $f_{B}$ could also be evaluated from the attenuation time of the oscillations $\tau$ as $\alpha=(v \tau)^{-1}$.

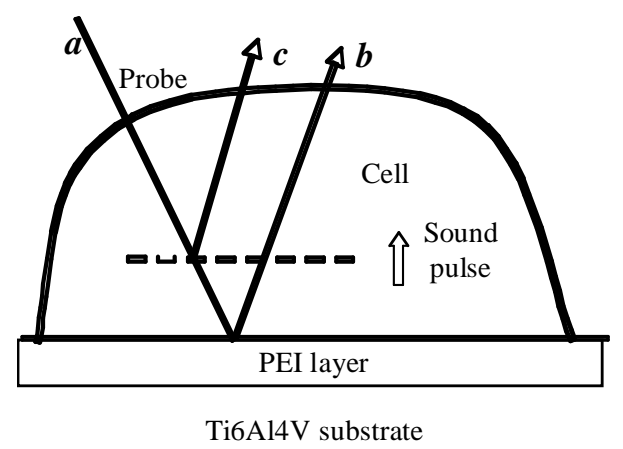

Figure1. Principle of the Brillouin scattering oscillations in the alive single cell. Laser pulses are labeled as following: incident $(a)$, reflected by the substrate $(b)$, scattered by the acoustic pulse $(c)$. 


\section{Results, in depth resolution and sensitivity to adhesion}

Typical signal of the reflectivity variation in a cell on a titanium alloy substrate is shown in the inset of Fig. 2. It consists mainly of Brillouin scattering oscillations superposed on a thermal decay due to the diffusion of the heat. The first peak is a consequence of the absorption of the pump pulse by the substrate. The thermal background is subtracted from the experimental signal (curve 1 in Fig. 2). The acoustic signal is detected also in an alive cell on a titanium alloy substrate after PEI deposition (curve 2 in Fig. 2). Several results are derived from the analysis of signals in Fig. 2.

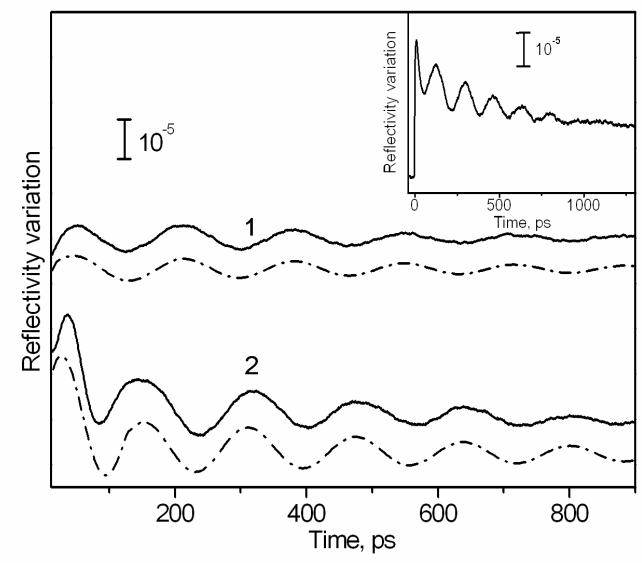

Figure 2. In inset: the total reflectivity variation signal measured in vitro in the vacuole of the cell on titanium alloy substrate without the polymer layer. Brillouin scattering oscillations in Allium Cepa alive single cell on titanium alloy substrates without (up) and with (down) polyethyleneimine (PEI) intermediate layer (experiment in solid lines, calculation in dashed lines). The measured frequencies and attenuation time of Brillouin scattering oscillations are $f_{B}=5.7 \pm 0.1 \mathrm{GHz}$ and $\tau=0.4 \pm 0.15 \mu \mathrm{s}$ in the cell and $f_{B}=7.5 \pm 0.1 \mathrm{GHz}$ in the PEI ( $\tau$ not measured) respectively.

First, fitting the experimental data with a sine function damped by an exponential decay, averaged values of the frequency and attenuation time of Brillouin scattering oscillations have been evaluated as $f_{B}=5.7 \pm 0.1 \mathrm{GHz}$ and $\tau=0.40 \pm 0.15 \mu \mathrm{s}$, respectively. These values are obtained as a result of the measurements of $f_{B}$ and $\tau$ at ten different locations inside the same vacuole. Similar periods and attenuation times are obtained either with or without the polymer coating. Choosing the optical index close to that in typical cells [4] as $n=1.4$, the averaged velocity of ultrasound in vacuole is obtained from (1) as $1.60 \pm 0.10 \mu \mathrm{m} / \mathrm{ns}$. Note that the sound velocity in water at this temperature of $22^{\circ} \mathrm{C}$ is equal to $1.49 \mu \mathrm{m} / \mathrm{ns}$ [5] which is different from the experimental value in vacuole. Similar increase of sound velocity has been observed in human keratinocyte cell (HaCaT) by $1 \mathrm{GHz}$ scanning acoustic microscopy [6]. It should be pointed out that the accurate evaluation of local value of the optical index of cell would be necessary for measurement of accurate value of sound velocity. The sound attenuation coefficient in vacuole at the frequency of Brillouin scattering is obtained from the attenuation time and sound velocity as $\alpha=1.5 \pm 0.1 \mu \mathrm{m}^{-1}$. Corresponding value of ultrasound penetration depth in the cell is $\alpha^{-1}=0.7 \mu \mathrm{m}$ which is significantly less than the typical thickness. For comparison, attenuation of $5.7 \mathrm{GHz}$ sound in water at $20^{\circ} \mathrm{C}$ provides an estimation of 
the corresponding penetration depth of $1.2 \mu \mathrm{m}$ [5]. Same increase of sound absorption in cell cytoplasm are observed in HaCaT cells at lower frequency [6].

Second, calculations of Brillouin scattering oscillations in the cell with and without interlayer have been performed according to the model described in Ref. 7. These calculations account for wave generation and reflectometric detection in the 3 layers structure (cell, PEI, and substrate). They allow estimation of the values of the physical properties of the polymer interlayer. Using the parameters measured previously for the cell, the best calculated fit of the experimental results is obtained with the following values for the polymer layer: $L=0.255 \mu \mathrm{m}$ (thickness), $v_{i}=2 \mu \mathrm{m} / \mathrm{ns} \rho_{I}=10^{3} \mathrm{~kg} / \mathrm{m}^{3}$, and $n=1.5$. These values are typical for polymers[8]. Very good description of phase and frequency of Brillouin scattering oscillations with/without the polymer is obtained with the mentioned parameters (Fig. 2). It is clear from Fig. 2 that the Brillouin frequencies in the PEI and the cell are different. When the acoustic wave generated in the $\mathrm{Ti}$ substrate, propagating through all the PEI layer, reaches the interface with the cell, the Brillouin frequency suddenly changes according to Eq. (1). The spatial depth resolution is then better than the thickness of the PEI layer $(0.25 \mu \mathrm{m})$. The change in frequency provides easy monitoring of both the longitudinal sound velocity and the thickness of the PEI intermediate layer. The 200 degrees phase shift of the Brillouin scattering oscillations in the cell, due to the polymer layer, is also correctly reproduced by the simulation. It is due to the delay of the sound pulse propagation in the PEI interlayer. Indeed, assuming a sound velocity in the polymer typically of $2 \mu \mathrm{m} / \mathrm{ns}$ provides a time delay of $0.12 \mathrm{~ns}$ which approximately corresponds to half a period of Brillouin oscillation in the cell vacuole

Third, Fig. 2 shows an increase of the oscillations amplitude of about 70\%, in the experiment with the interlayer. The magnification of wave amplitude provided by the acoustic matching caused by the polymer layer can be calculated using the physical parameters estimated above. An improvement of the photoacoustic signal of $30 \%$ only is predicted. The additional magnification observed in the second experiment could suggest that adhesion of the cell on the titanium alloy substrate or on the polymer coating is different. Indeed, cell adhesion is a complicated and intricate phenomenon related to the geometrical, physical, chemical properties of the surface such as roughness, porosity, surface tension, etc. This detailed study is beyond the scope of this paper.

\section{Reproducibility and sensitivity}

To illustrate the reproducibility and the sensitivity of the method, experiments have been achieved on two sets, each including ten cells, of yellow and white allium cepa cells. The measured frequencies and phases of the Brillouin oscillations measured in cells vacuole and cells kernel are shown in Figure 3. Owing to the similarities of the mechanical properties, results measured in the cytoplasm of the two allium cepa cells varieties are equal. In addition, the Brillouin frequencies measured in the kernel of either yellow or white allium cepa cells are sensitively different. 

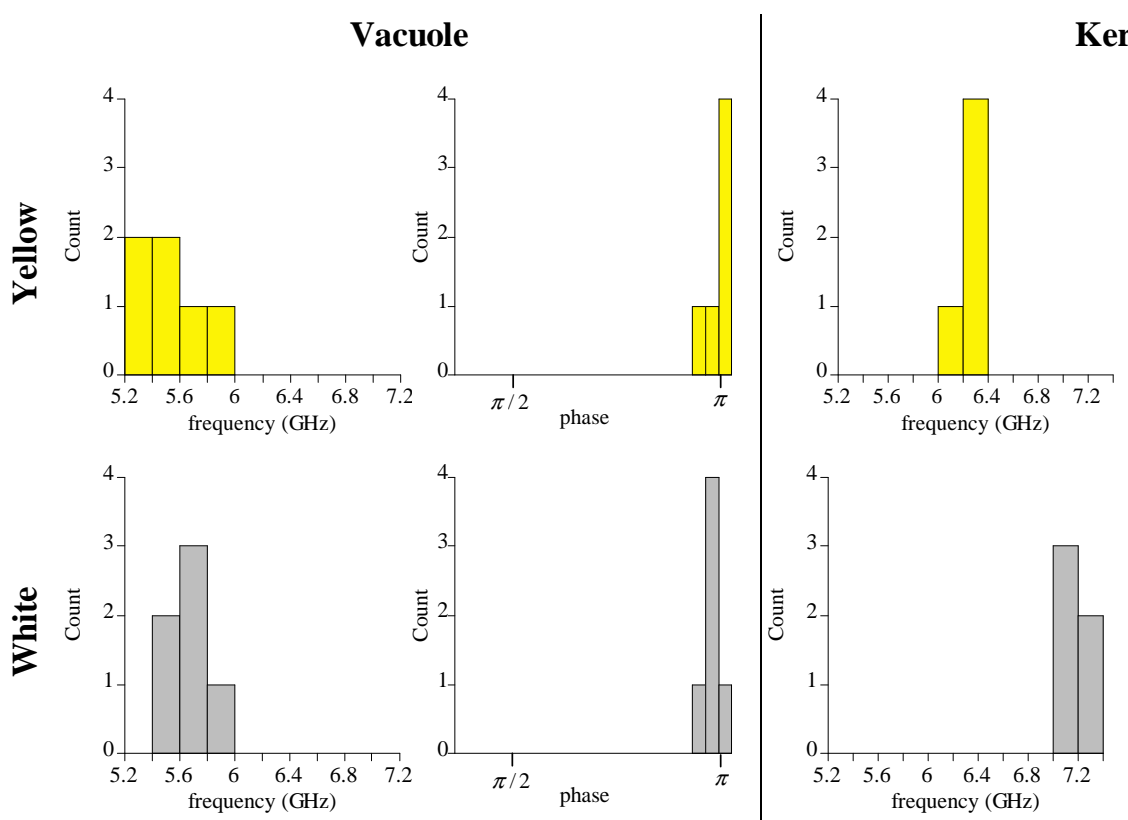

Kernel
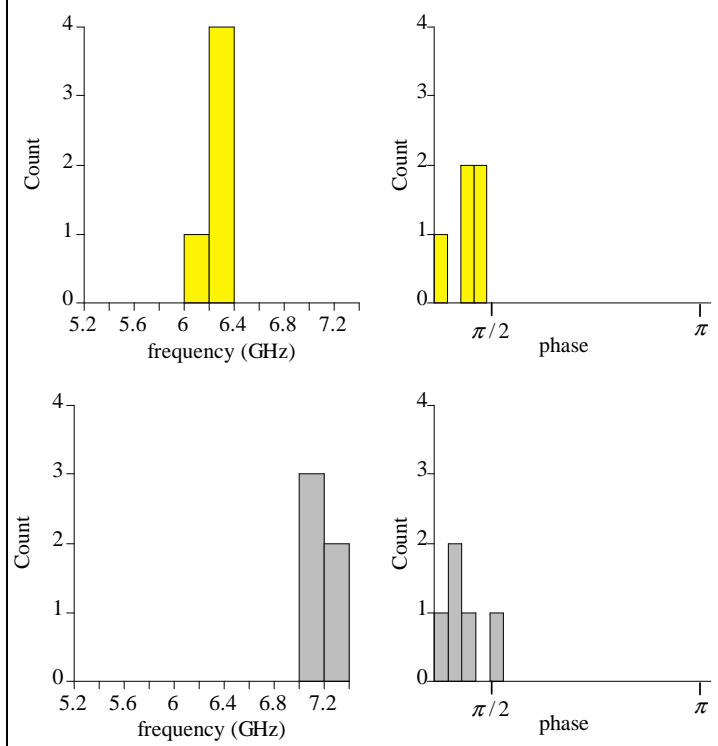

Figure 3. frequencies and phases of the Brillouin oscillations measured in cells vacuole and cells kernel for two sets, each including ten cells, of yellow and white allium cepa cells.

\section{Conclusions}

Very promising abilities of the picosecond ultrasonic technique for the non-invasive study of biological alive single cells have been demonstrated in this paper. The application of contactless and very high frequency picosecond ultrasonic technique will improve significantly the space resolution of acoustic imaging of alive cells. It will also provide more information about mechanical properties of the cell. Values of the longitudinal sound velocity $1.6 \pm 0.1 \mu \mathrm{m} / \mathrm{ns}$ and attenuation, $1.5 \pm 0.1 \mu \mathrm{m}^{-1}$ of $5.7 \pm 0.1 \mathrm{GHz}$ acoustic waves in a vegetal cell vacuole have been obtained in vitro. From the velocity, cell compressibility nearby the cell-substrate interface can now be mapped with a resolution better than $2 \mu \mathrm{m}$ laterally and $0.25 \mu \mathrm{m}$ in depth. In addition to single cell elastography, the sensitivity of the measurements to cell adhesion suggests promising perspectives for the imaging of the physiological cell functions or the cell in function of its surroundings or its health.

\section{References}

1. C. Thomsen, J. Strait, Z. Vardeny, H.J. Maris. J. Tauc, J.J. Hauser, "Coherent phonon generation and detection by picosecond light pulses", Phys. Rev. Lett., 53, pp 989, 1984.

2. P.-L. Kuo, S. K. Ghosh, W.-J. Liang, Y.-T. Hsien, "Hyperbranched polyethyleneimine architecture onto poly(allylamine) by simple synthetic approach and the chelating characters", Journal of Polymer Science: Part A: Polymer Chemistry, 39, pp 3018, 2001.

3. L.J. Shelton, F. Yang, W.K. Ford, H.J. Maris, "Picosecond ultrasonic measurement of the velocity of phonons in water", Phys. Stat. Sol. (B), 242, pp 1379, 2005. 
5. V.A. Grosso, C.W. Mader "Speed of Sound in Pure Water", J. Acoust. Soc. Am., 52, pp 1442, 1972.

4. J. Beuthan, O. Minet, J. Helfmann, M. Herrig, and G. Muller, "The spatial variation of the refractive index in biological cells", Physics Med. Biol., 41, pp 369, 1996.

6. T. Kundu, J.P. Lee, C. Blase, and J. Bereiter-Hann, "Acoustic microscope lens modeling and its application in determining biological cell properties from singleand multi-layered cell models", J. Acoust. Soc. Am., 120, pp 1646, 2006.

7. C. Rossignol, B. Perrin, S. Laborde, L. Vandenbulcke, M.I. De Barros, and P. Djemia, "Nondestructive evaluation of micrometric diamond films with an interferometric picosecond ultrasonics technique", J. Appl. Phys., 95, pp 4157, 2004.

8. A.G. Every, A. McCardy, "Numerical data and fundamental relationships in science and technology", New Series, Group III of Landolt-Bornstein, vol. 29A, Springer Berlin (1992). 